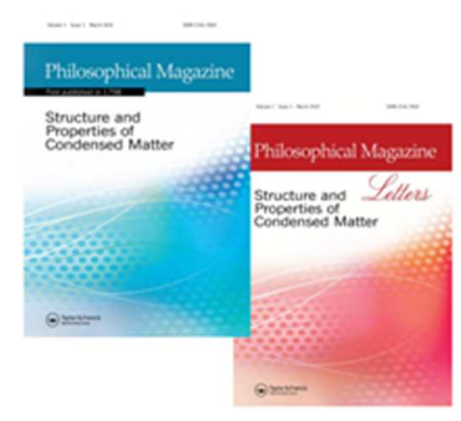

\title{
Berkovich nanoindentation study of monocrystalline tungsten: A crystal plasticity study of surface pile-up deformation
}

\begin{tabular}{|r|l|}
\hline Journal: & Philosophical Magazine \& Philosophical Magazine Letters \\
\hline Manuscript ID & TPHM-16-Jul-0328.R1 \\
\hline Journal Selection: & Philosophical Magazine \\
\hline Date Submitted by the Author: & $09-J a n-2017$ \\
\hline Complete List of Authors: & $\begin{array}{l}\text { Yao, Weizhi; Institute of Materials, China Academy of Engineering Physics, } \\
\text { You, Jeong-Ha; Max-Planck-Institut fur Plasmaphysik }\end{array}$ \\
\hline Keywords: & nanoindentation, tungsten, finite-element modelling, plastic deformation \\
\hline Keywords (user supplied): & crystal plasticity, pile-up \\
\hline & \multicolumn{2}{|l}{} \\
\hline
\end{tabular}

SCHOLARONE ${ }^{\mathrm{m}}$

Manuscripts 


\title{
Berkovich nanoindentation study of monocrystalline tungsten: A crystal plasticity study of surface pile-up deformation
}

\author{
W.Z. Yao ${ }^{\mathrm{a}}$, J.H. You ${ }^{\mathrm{b}}$ \\ ${ }^{a}$ Institute of Materials, China Academy of Engineering Physics, Jiangyou, PR China \\ ${ }^{b}$ Max-Planck-Institut für Plasmaphysik, Boltzmanstr.2, Garching, Germany \\ corresponding author W.Z.Yao e-mail: weizhi.yao@yahoo.com
}




\title{
Berkovich nanoindentation study of monocrystalline tungsten: A crystal plasticity study of surface pile-up deformation
}

\begin{abstract}
In this paper, it was investigated whether Berkovich indentation test with a triangular-based pyramidal imprint would exhibit the same surface pile-up deformation behavior as in Vickers or spherical indentation tests. The characteristic correlation between the pile-up patterns of monocrystalline tungsten and the geometry of slip systems was examined both experimentally and computationally. Surface pile-up patterns for three different crystallographic orientations of specimens with corresponding rotational crystal symmetry were characterized. In addition, the effect of the varying azimuthal orientation of the indenter on the pile-up patterns was also discussed. Predictions from finite element simulation based on the crystal plasticity theory are also presented and compared with the measured results. It was found that the surface pile-up patterns of Berkovich indentation did not necessarily reflect the rotational crystal symmetry of tungsten single crystal specimens. The pile-up patterns were affected by the variation of the indenter's azimuthal orientation. The height of the pile-up hillocks was often highly non-uniform even on the same surface plane indicating strong influence of slip geometry leading to the plastic anisotropy.
\end{abstract}

Keywords: crystal plasticity; nanoindentation; tungsten; finite-element modelling; pile-up; plastic deformation

\section{Introduction}

Nanoindentation has been used as an effective tool for measuring various kinds of mechanical properties of solids on nano- and microscopic scales. For instance, the measurement of load-penetration curves or the examination of the plastic imprint produced by an instrumented indentation test enables one to extract the elastic and plastic properties of the material such as elastic modulus, yield stress, work hardening rate, etc [1-4]. To this end, analytical models or numerical simulations are used for calibrating material parameters. Here, mechanical properties are often assumed to be 
isotropic for the sake of simplicity. However, a nanoindentation test, even in the case of a polycrystalline material, is likely to produce an imprint within a single grain, where the deformation by indentation can take place in a highly anisotropic manner. In the literature several works can be found devoted to the analytical description of indentation behavior of mono-crystalline materials [5-9].

The surface topography produced by indentation loads may exhibit either upward (pile-up) or downward (sink-in) deformation around the imprint with respect to the original surface plane $[10,11]$. In the case of isotropic materials, whether the indented surface undergoes pile-up or sink-in deformation is primarily determined by strainhardening capability [12]. When single crystals are subjected to an indentation load, the geometrical relationship between the loading axis and the slip systems come into play. It is obvious that a spherical (or conical) indenter can best detect the plastic anisotropy of single crystals owing to the axisymmetric contact stress field [13-16]. A previous spherical indentation study on monocrystalline tungsten showed that discrete pile-up patterns were produced reflecting the crystal symmetry and the slip system geometry (note that tungsten crystal is fully isotropic in elastic response)[13].

On the contrary, polygonal indenters (Vickers or Berkovich) are normally used to measure elastic moduli or hardness[17], load-displacement curves [18, 19] or incipient yield [20-23]. Sometimes, however, Vickers indenter was used to characterize the surface pile-up patterns of FCC and BCC single crystals. Peralta et al. [24], Stelmashenko et al. [25] and Eidel [12] observed that the pile-up patterns produced by Vickers indentation test were significantly affected by the geometric feature of the slip systems, but hardly by the azimuthal orientation of the indenter. Regarding the Berkovich indentation test, there is no related report in the literature. Now, a question arises as to whether a Berkovich indenter with a triangular-based pyramidal tip would 
have the same effect on the pile-up deformation as a Vickers indenter with a squarebased pyramidal tip does. This is not a trivial question, as the 3 -fold rotational symmetry of the indenter tip shape does not coincide with the rotational symmetry of cubic crystal planes with low Miller indices, and thus, because the inherent plastic anisotropy is obscured by the triangular geometry of Berkovich indentation.

The aim of the current work was to investigate the characteristics of plastic yield behavior of monocrystalline tungsten under Berkovich indentation tests in terms of crystal symmetry and the slip system geometry. To this end, the surface pile-up patterns on different specimen planes with the 2-, 3- and 4-fold rotational crystal symmetry were examined. In addition, the effect of the azimuthal orientation of the indenter on the pileup patterns was also investigated.

\section{Experiment}

\subsection{Specimen preparation}

Commercial products of single crystals of tungsten with purity of $99.99 \%$ (Goodfellow $\left.{ }^{\circledR}\right)$ were used for the nanoindentation tests. The as-delivered crystals had a cylindrical shape with a diameter of either $9 \mathrm{~mm}$ or $6 \mathrm{~mm}$ and a length of about $20 \mathrm{~mm}$. The longitudinal axis of the tungsten rods was aligned to one of the following three crystal orientations, namely, [100], [110] and [111], respectively. The single crystal rods were cut into thin disc-shaped slices with a thickness of about $1 \mathrm{~mm}$ using a diamond wire saw. After each cutting operation the crystal orientation was checked by means of X-ray diffraction (Laue method) and the orientation error was controlled within $2 \%$. The cut surfaces were at first mechanically polished and subsequently electrochemically polished in $1.5 \% \mathrm{NaOH}$ electrolyte at $25 \mathrm{~V}$ for $5 \mathrm{~min}$ in order to get rid of any damaged or deformed layer that was possibly produced by the mechanical 
polishing. After the electrochemical treatment the surface roughness of the specimens was significantly reduced where the peaks of surface profile was measured to range between 3-10 $\mathrm{nm}$ by the atomic force microscope (AFM).

\subsection{Nanoindentation tests}

Nanoindentation tests were conducted at room temperature using an instrumented nano\& microindentation testing device (Micro Materials Ltd. UK). A diamond Berkovich indenter was used. The area function of the indenter as well as the machine compliance was calibrated prior to the measurements using the procedure by Oliver and Pharr [26]. The maximum penetration depth was kept at about $\delta \approx 1 \mu \mathrm{m}$ and the loading rate was fixed at $0.5 \mathrm{mN} / \mathrm{s}$. For acceptable statistical significance testing was repeated thirty times for each specimen. In addition, for each loading case, indentation test was repeated on two different specimens that have the same crystal axis orientation.

It should be emphasized that the final depth $1 \mu \mathrm{m}$ is large enough to avoid major effect from indentation size effect (see for example [27]). This has also been validated by repeated nanoindentation tests with maximum penetration depth ranging from several tens of nanometers to $1 \mu \mathrm{m}$ [23]. The indentation hardness was about $4.5 \mathrm{GPa}$ and remained rather constant when the maximum indentation depth was larger than 600 nm. Our experiment and simulation were restricted to this length scale since the constitutive law used for simulation didn't include length scale parameters and indentation size effect cannot therefore be reproduced by simulations.

\subsection{Surface analysis}

AFM was applied to map the surface topography after nanoindentation. The imprint formed by a Berkovich indenter produced an equilateral triangle with an edge length of $7 \mu \mathrm{m}$ at a depth of $1 \mu \mathrm{m}$ approximately. 
An area of $15 \times 15 \mu \mathrm{m}^{2}$ including an imprint was scanned by AFM to measure the surface contour of pile-up patterns with high precision. These experimental data of surface deformation were then compared with the simulation results.

In order to measure the crystallographic relationships between the crystal orientation and the pile-up patterns, electron backscatter diffraction (EBSD) technique was employed together with scanning electron microscopy (SEM). Here, EBSD allowed crystallographic mapping along the azimuthal orientation around the imprints. By combining the data obtained from the EBSD and AFM measurements, detailed information on the pile-up deformation could be extracted from the nanoindentation tests and compared with the predictions of simulation.

\section{Simulation}

\subsection{Theoretical background: crystal plasticity}

The crystal plasticity model is capable of describing plastic deformation processes by taking account dislocations gliding along activated slip systems, which are determined by two vectors, the unit normal to the slip plane $m$ and the unit vector $s$ in the slip plane along the slip direction. The mathematical formulation of the kinematics of the crystal plasticity model used in this paper is from Peirce et al [28-30]. Only a brief overview of it will be presented here. More details about formulating of elastic-plastic single crystal deformation refer to the general framework of Hill [31, 32] and Rice [33].

A mathematical description of the kinetics and constitutive relations usually starts with the computation of the velocity gradient $L(=\partial v / \partial x)$ which can be expressed as

$$
L=\dot{F} \cdot F^{-1}
$$

where $F$ is the total deformation gradient, and it can be decomposed into 


$$
F=F^{*} \cdot F^{p}
$$

where $F^{p}$ denotes plastic shear deformation of the material caused by slip motion of its active slip systems, and where $F^{*}$ denotes deformation gradients due to both elastic stretching and rotation of the lattice. It is assumed that the elastic properties are unaffected by plastic slip. The plastic deformation $F^{p}$ is described by

$$
\dot{F}^{p}=L^{p} \cdot F^{p}
$$

with $L^{p}$ being the plastic flow rate, which can be determined by

$$
L^{p}=\sum_{\alpha} \dot{\gamma}^{\alpha} s^{\alpha} m^{\alpha}
$$

where $\dot{\gamma}^{\alpha}$ is the shearing rate of slip system $\alpha$, and unit vectors $s^{\alpha}$ and $m^{\alpha}$ are the slip direction and normal to slip plane in the reference configuration, respectively. The shearing rate on each slip system depends on the resolved shear stress $\tau^{\alpha}$ and the strength of that slip system $g^{\alpha}$ and it is formulated as

$$
\dot{\gamma}^{\alpha}=\dot{a}^{\alpha}\left|\frac{\tau^{\alpha}}{g^{\alpha}}\right|^{n} \operatorname{sign}\left(\tau^{\alpha}\right),
$$

in which the constant $\dot{a}^{\alpha}$ is the reference value of the strain rate on slip system $\alpha$ and $n$ is the strain rate sensitivity exponent.

The strain hardening is determined by the following evolution law:

$$
\dot{g}^{\alpha}=\sum_{\beta} h_{\alpha \beta} \dot{\gamma}^{\beta}
$$

where $h_{\alpha \beta}$ are the slip hardening matrix. The diagonal terms $h_{\alpha \alpha}$ indicate selfhardening on slip system $\alpha$, whereas the off-diagonal entries $h_{\alpha \beta}(\alpha \neq \beta)$ stand for latent hardening of the system $\alpha$. The summation ranges over all activated slip systems. The self-hardening moduli formed by Peirce, Asaro and Needleman [28] as 


$$
h_{\alpha \alpha}=h(\gamma)=h_{0} \operatorname{sech}^{2}\left|\frac{h_{0} \gamma}{\tau_{s}-\tau_{0}}\right| \text {, }
$$

where $h_{0}$ is the initial hardening modulus, $\tau_{0}$ is for the initial strength of slip system, $\tau_{s}$ is the saturation strength, and $\gamma$ is the cumulative shear strain on all slip systems:

$$
\gamma=\sum_{\alpha} \int_{0}^{t}\left|\dot{\gamma}^{\alpha}\right| \mathrm{d} t
$$

The latent hardening moduli are given by

$$
h_{\alpha \beta}=q h(\gamma) \quad(\alpha \neq \beta)
$$

where $q(1 \leq q \leq 1.4)$ is the latent hardening parameter describing the ratio of noncoplanar hardening to coplanar hardening.

\subsection{Finite element modeling and simulation of indentation}

In this study a commercial FEM code ABAQUS was used together with a user-defined material subroutine (UMAT) where the theoretical model of crystal plasticity described in the previous section was implemented by Huang [34]. This crystal plasticity model had been applied to study the evolution of texture during tungsten wire drawing[35] and characterize the fracture toughness of single-crystalline tungsten using notched microcantilever specimens[36].

The geometry of the Berkovich indenter was modeled as a rigid three-sided pyramid having an inclination angle of $65.27^{\circ}$. As the symmetry of the indenter did not coincide with the crystal's symmetry, the whole shape of the indenter and the full surface plane had to be taken into account as a three-dimensional model. The indented specimen was modeled as a cylinder of radius $r=40 \mu \mathrm{m}$ and thickness $h=40 \mu \mathrm{m}$ as illustrated in Fig. 1. 
The cylindrical specimen was discretized with the linear brick elements (C3D8R) and wedge elements (C3D6) while the indenter was assumed to be a rigid body meshed with R3D4 and R3D3 elements. The meshing was done on the 24 circumferential sectors of the cylinder with angular partition of $15^{\circ}$ each. The mesh model of the cylinder consisted of 12576 elements and 12965 nodes. For accuracy, mesh was refined near the contact area. The size of the smallest element was about $0.08 \mu \mathrm{m}$ and $0.31 \mu \mathrm{m}$ in hoop and radial direction, respectively.

In Fig. 1 load was applied downward along the $y$-axis of the global coordinate system where the nodal displacement on the bottom surface were fixed in the $y$-axis and the hoop direction. The vertical displacement of the indenter was limited up to $\delta \approx 1 \mu \mathrm{m}$ at the maximum which was regarded as being large enough to overwhelm any local surface effect in both experiment and simulation.

Friction between the indenter and the material is known to affect the height of pileups but hardly the load-displacement curves [14]. Unfortunately, there is no literature data on the friction coefficient for the given material system. To determin an appropriate value of friction coefficient for the present FEM model, a parametric simulation was made with four different values of friction coefficient, namely $0,0.1,0.2$, and 0.3 . The result showed that the maximum pile-up height was decreased by about $7 \%$ when the friction coefficient was increased from 0 to 0.3 ( ( $\left.\begin{array}{lll}1 & 0 & 0\end{array}\right)$ plane was indented) indicating that friction would have only a minor effect on pile-up deformation. Here, a friction coefficient of 0.1 was assumed.

For the indentation simulation on the $(100)$ plane, we considered three distinct cases of azimuthal orientation of the Berkovich indenter, $\theta=-20^{\circ}, 38^{\circ}, 45^{\circ}$ where $\theta$ is the angle between the [0 01 1] direction and one edge of the imprint as illustrated in Fig. 2. For the indentation simulation on the (110) and (111) planes, again three different 
cases of azimuthal orientation of the indenter were considered (not shown for the sake of brevity). According to the AFM and EBSD measurements of the imprints after nanoindentation test, the azimuthal orientations of the indenter were $-90^{\circ},-45^{\circ}$ and $60^{\circ}$ for the (110) plane and $-100^{\circ}, 60^{\circ}$ and $110^{\circ}$ for the (111) plane, respectively.
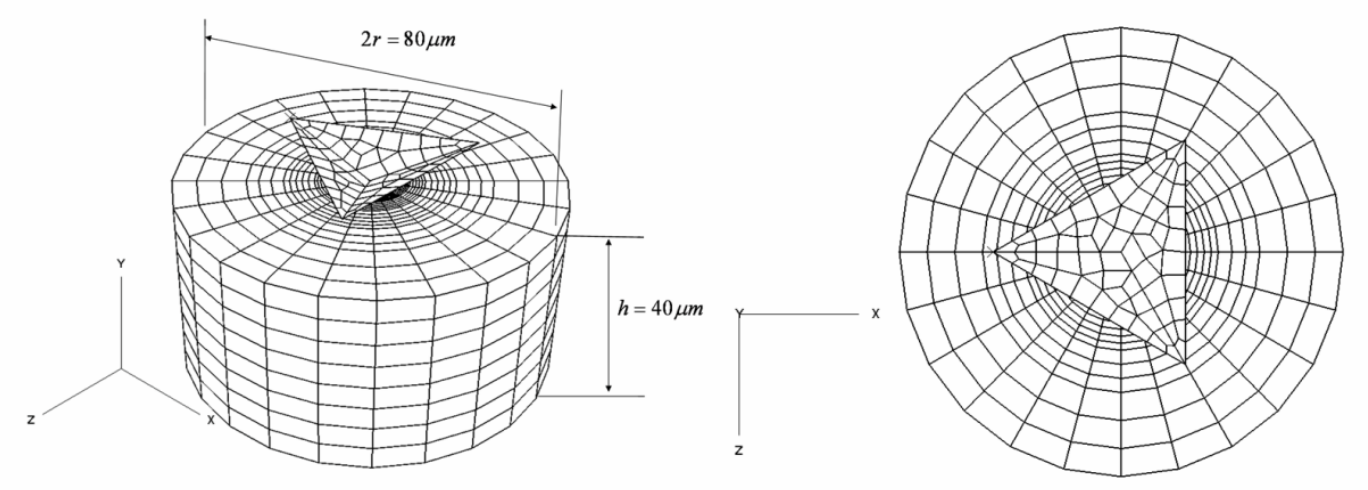

Fig. 1. Three-dimensional finite element model for Berkovich indentation (isometric and top view).

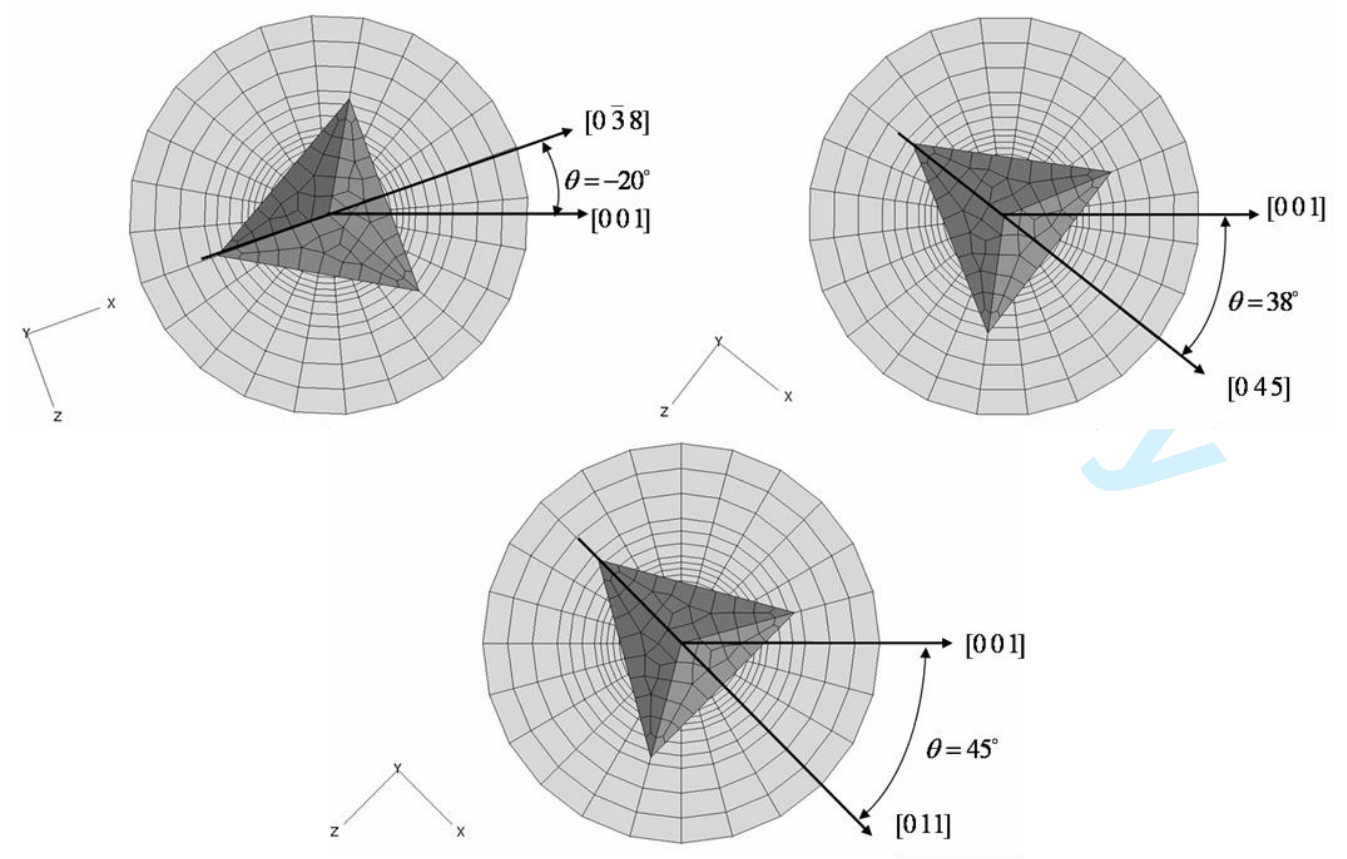




\subsection{Calibration of material parameters}

The purpose of this section is to determine the input parameters for our FEM. However, it is very difficult and perhaps not practical to determine the entire 20 unknown constants (when all three slip system families of tungsten crystal are activated, see table 1) which would be needed to define the elastic-plastic behavior of single crystal materials, since their identification requires reverse techniques, in which extensive numerical simulations are necessary. Instead, attention is focused on extracting the key parameters of the material model, as well as taking advantages of well-known conclusions from literatures to reduce the number of unknown parameters. The key material parameters of the crystal plasticity theory considered here are as follows:

- Two elastic constants for all slip systems: $E$ (modulus) and $v$ (Poisson's ratio)

The elastic constants of a tungsten crystal are known to be fully isotropic $[37,38]$ and have the values of $400 \mathrm{GPa}$ and 0.28 for $\mathrm{E}$ and $v$, respectively $[38,39]$.

- Two viscous parameters: $n$ (strain rate sensitivity exponent) and $\dot{a}$ (reference shear strain rate)

These two viscous parameters are set to 20 and $0.001 \mathrm{~s}^{-1}$ and assumed constant in all simulations since the primary meaning of the strain rate sensitivity exponent and the reference shear rate is of a numerical nature rather than being related to the micromechanical properties[15].

- Four plastic parameters: $h_{0}$ (initial hardening modulus), $\tau_{s}$ (saturation stress), $\tau_{0}$ (initial yield stress) and $q$ (latent hardening parameter). 
Bcc materials have three slip system families containing 48 slip systems (Table 1). At room temperature slip system family I $\{110\}<111>$ and family II $\{112\}<111>$ are the commonly active slip system families[40-42]. Therefore only these two slip system families were taken into account in our simulations, then two groups of plastic parameters for these two activated slip system families had to be determined which count up to 8 constants. In the further process to reduce the total number of independent material variables, an assumption was made a priori: the plastic parameters $h_{0}, \tau_{s}$, and $\tau_{0}$ for the two slip system families were selected to satisfy the ratio of $1: 1.2$, e.g. the initial yield stresses for the slip families were selected to satisfy $\tau_{0}^{\mathrm{I}}<\tau_{0}^{\mathrm{II}}$ with following ratio, 1:1.2, Similar ratio had been applied to simulate $\mathrm{W}$ wire drawing process [35]. Remembering that the experimental data [43] showing that the ratio of the resolved shear yield stresses measured for the slip systems $\{110\}<111>$ and $\{112\}<111>$ was roughly $1: 1.2$.

Table 1 Slip systems of bcc crystals

\begin{tabular}{|c|c|c|c|c|c|c|c|}
\hline \multicolumn{2}{|c|}{$\begin{array}{l}\text { Family I } \\
\{110\}<111>\end{array}$} & \multicolumn{2}{|c|}{$\begin{array}{l}\text { Family II } \\
\{112\}<111>\end{array}$} & \multicolumn{4}{|c|}{$\begin{array}{l}\text { Family III } \\
\{123\}<111>\end{array}$} \\
\hline (1) & $\overline{(110)[1 \overline{1} 1]}$ & (1) & $(112)[11 \overline{1}]$ & (1) & $\overline{(123)[11 \overline{1}]}$ & (13) & $\overline{(132) \text { [1]1] }}$ \\
\hline (2) & $(110)[\overline{1} 11]$ & (2) & $(\overline{1} 12)[1 \overline{1} 1]$ & (2) & $(\overline{1} 23)[1 \overline{1} 1]$ & (14) & $(\overline{1} 32)[11 \overline{1}]$ \\
\hline (3) & $(101)[\overline{1} 11]$ & (3) & $(1 \overline{1} 2)[\overline{1} 11]$ & (3) & $(1 \overline{2} 3)[\overline{1} 11]$ & (15) & $(1 \overline{3} 2)[111]$ \\
\hline (4) & $(101)[11 \overline{1}]$ & (4) & $(11 \overline{2})[111]$ & (4) & $(12 \overline{3})[111]$ & (16) & $(13 \overline{2})[\overline{1} 11]$ \\
\hline (5) & $(011)[1 \overline{1} 1]$ & (5) & $(121)[1 \overline{1} 1]$ & (5) & $(312)[\overline{1} 11]$ & (17) & $(213)[11 \overline{1}]$ \\
\hline (6) & $(011)[11 \overline{1}]$ & (6) & $(\overline{1} 21)[11 \overline{1}]$ & (6) & $(\overline{3} 12)[111]$ & (18) & $(\overline{2} 13)[1 \overline{1} 1]$ \\
\hline$(7)$ & $(\overline{1} 10)[111]$ & (7) & $(1 \overline{2} 1)[111]$ & (7) & $(3 \overline{1} 2)[11 \overline{1}]$ & (19) & $(2 \overline{1} 3)[\overline{1} 11]$ \\
\hline (8) & $(\overline{1} 10)[11 \overline{1}]$ & (8) & $(12 \overline{1})[\overline{1} 11]$ & (8) & $(31 \overline{2})[1 \overline{1} 1]$ & (20) & $(21 \overline{3})[111]$ \\
\hline (9) & $(10 \overline{1})[111]$ & (9) & $(211)[\overline{1} 11]$ & (9) & $(231)[1 \overline{1} 1]$ & (21) & $(321)[\overline{1} 11]$ \\
\hline (10) & $(10 \overline{1})[1 \overline{1} 1]$ & (10) & $(\overline{2} 11)[111]$ & (10) & $(\overline{2} 31)[11 \overline{1}]$ & (22) & $(\overline{3} 21)[111]$ \\
\hline (11) & $(01 \overline{1})[111]$ & (11) & $(2 \overline{1} 1)[11 \overline{1}]$ & (11) & $(2 \overline{3} 1)[111]$ & (23) & $(3 \overline{2} 1)[11 \overline{1}]$ \\
\hline (12) & $(01 \overline{1})[\overline{1} 11]$ & (12) & $(21 \overline{1})[1 \overline{1} 1]$ & (12) & $(23 \overline{1})[\overline{1} 11]$ & (24) & $(32 \overline{1})[1 \overline{1} 1]$ \\
\hline
\end{tabular}


Parametric studies showed that the latent hardening parameters $q$ had minor effect on the load-displacement curves and the pile-up patterns; therefore its value $q=1$ was taken from literature[28]. The three inelastic parameters, the initial hardening modulus $h_{0}$, the initial yield stress $\tau_{0}$ and the saturation stress $\tau_{s}$, were then determined by fitting the numerical load-displacement curves with experimental curves obtained from indentation tests performed on three crystallographic planes of SCW. After many trial and error tests, one set of the plastic parameters: $h_{0}=1350 \mathrm{MPa}, \tau_{0}=210 \mathrm{MPa}$, and $\tau_{s}$ $=530 \mathrm{MPa}$ for slip system family I was obtained. It was demonstrated that this parameter set could reproduce the load-displacement curves fairly well for three different cases of crystal orientation as shown in Fig. 3.
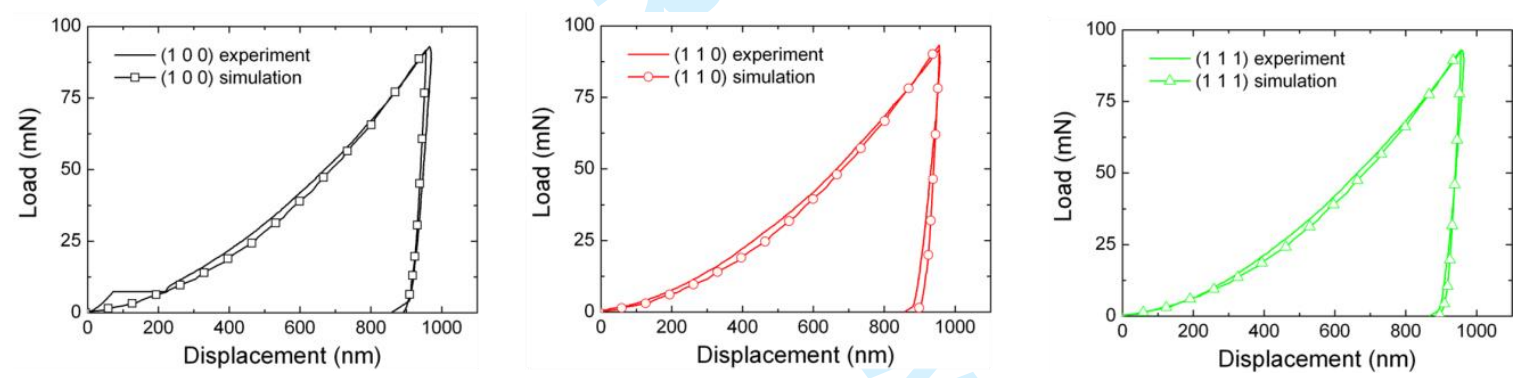

Fig. 3.Comparison of the experimental and CPFE simulated load-displacement curves from nanoindentation on SCW with different crystallographic planes: (100), (110), and (111).

\subsection{Analysis of the numerical nanoindentation results}

Fig. 4. shows an example in the top view (with indented (100) plane) of the plastic deformation after load was removed. One can observe the numerical results of the accumulative plastic shear strain on each slip system and the total accumulative plastic shear strain on all slip systems after unloading. It is shown that all slip systems are nonuniformly activated around the indentation imprint, and the reason could be that the 
Berkovich indenter imposes a complex stress field under the indenter in the material due to its particular geometry. Unfortunately, this kind of information cannot be directly compared to the experimental results such as surface topography. Therefore, only experimental and numerical surface deformation profiles will be compared.

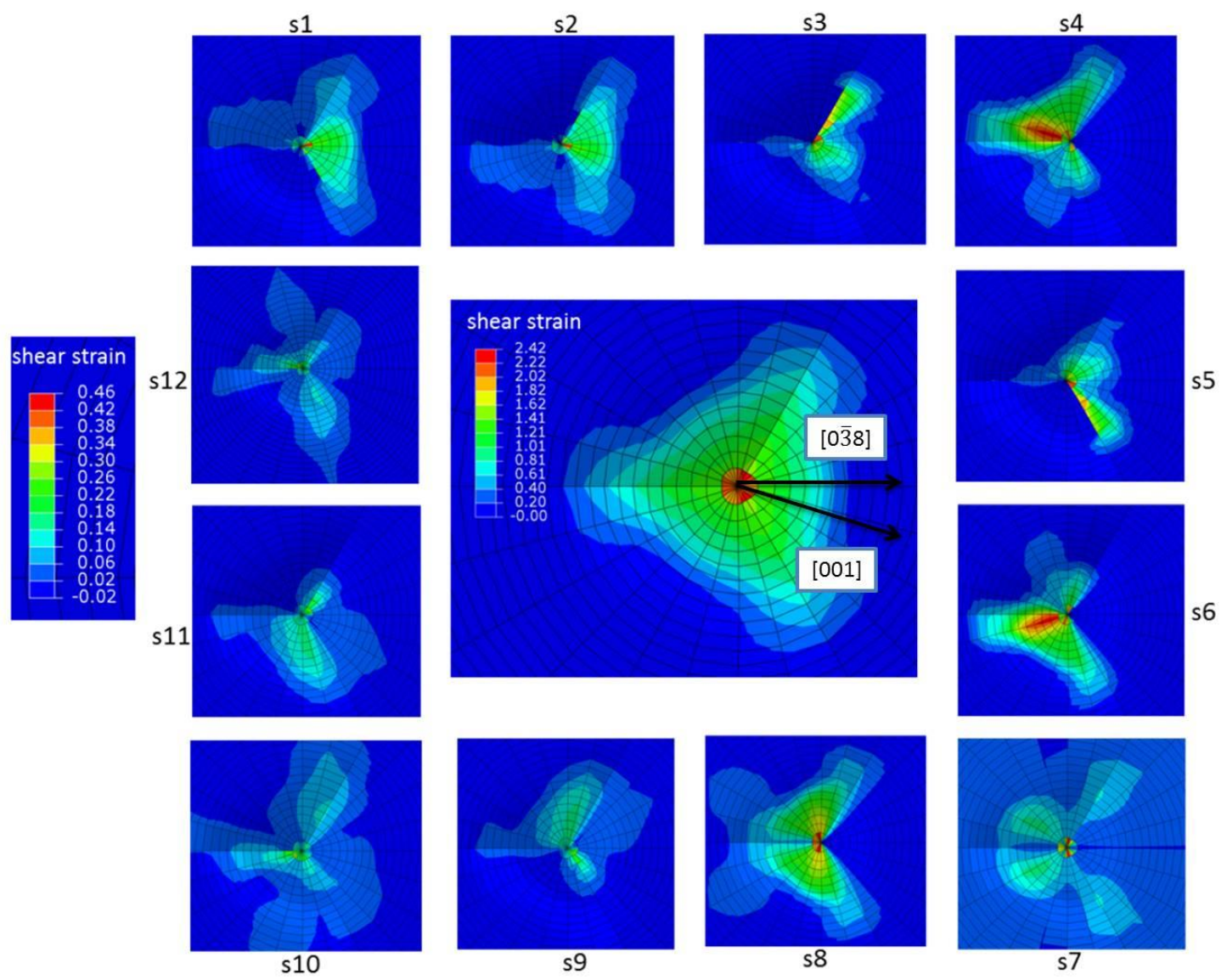

Fig. 4. Details of a (100) nanoindentation shear strain with in-plane rotated indenter $\theta=-20^{\circ}$ obtained in the top view: the total accumulative plastic shear strain (at the center of the figure), and the ones obtained on the 12 slip systems (slip system family I as shown in Table 1) after unloading.

\section{Results and discussion}

\subsection{Impact of crystal orientation on pile-up deformation}

The nanoindentation tests were carried out on tungsten surfaces each with one of the three different crystallographic planes, namely (100), (110) and (111). The contour 
profiles (height) of the surface pile-ups around the indented imprints measured by AFM scan are presented in Fig.5-Fig.7 (a-c), where each plot represents the respective map of one of the nine combinations between the three surface normal orientations and the three azimuthal orientations. For comparison, the simulation results are also given in Fig.5-Fig.7 ( $\left.\mathrm{a}^{*}-\mathrm{c}^{*}\right)$ below the corresponding experimental data.

The simulated surface pile-up contour maps exhibit at least a qualitatively good agreement with those of the measured deformation patterns for the most of the orientation cases, although the height of the hillocks tend to be notably underestimated in the predicted maps. The pile-up patterns and their maximum height seem to significantly depend on the orientation relationship between the specimens and the indenter. For example, the pile-up height reached $24-28 \%$ of the penetration depth on the (100) plane, $11-18 \%$ on the (110) plane and $12-16 \%$ on the (111) plane. In all indentation cases three hillocks were formed on each side of the triangular imprints, where the height of the pile-ups generally differed from each other.

In the previous micro-indentation study of monocrystalline tungsten that was performed with a spherical indenter, it was shown that the characteristic pile-up patterns directly reflected the crystal symmetry and the orientation relationship between the surface normal and the load axis $[13,44]$. Although the spherical indenter tip generates circularly symmetric contact loads (and thus such stress fields), exclusively discrete pile-up patterns were produced indicating an obvious evidence of plastic anisotropy due to discrete slip mechanisms (note that tungsten crystal is fully isotropic in elastic response).

On the contrary, the slip-induced plastic anisotropy is obscured in the Berkovich indentation by the polygonal shape of the imprints making the interpretation of the deformation patterns more complicated compared to the ball indentation case. The 
three-fold rotational symmetry of the Berkovich indenter coincides only with the crystal symmetry on the (111) plane while mismatches with that of the (100) and (110) planes. On the other hand, the broken circular symmetry of contact loads produced in the Berkovich indentation enables one to detect the effect of azimuthal orientation relative to the polar orientation of the indenter. Fig.5-Fig.7 (a-c) indeed reveals that the pile-up contour patterns have clear dependence on the azimuthal orientation of the indenter for a given crystal orientation as well as on the surface normal orientation.
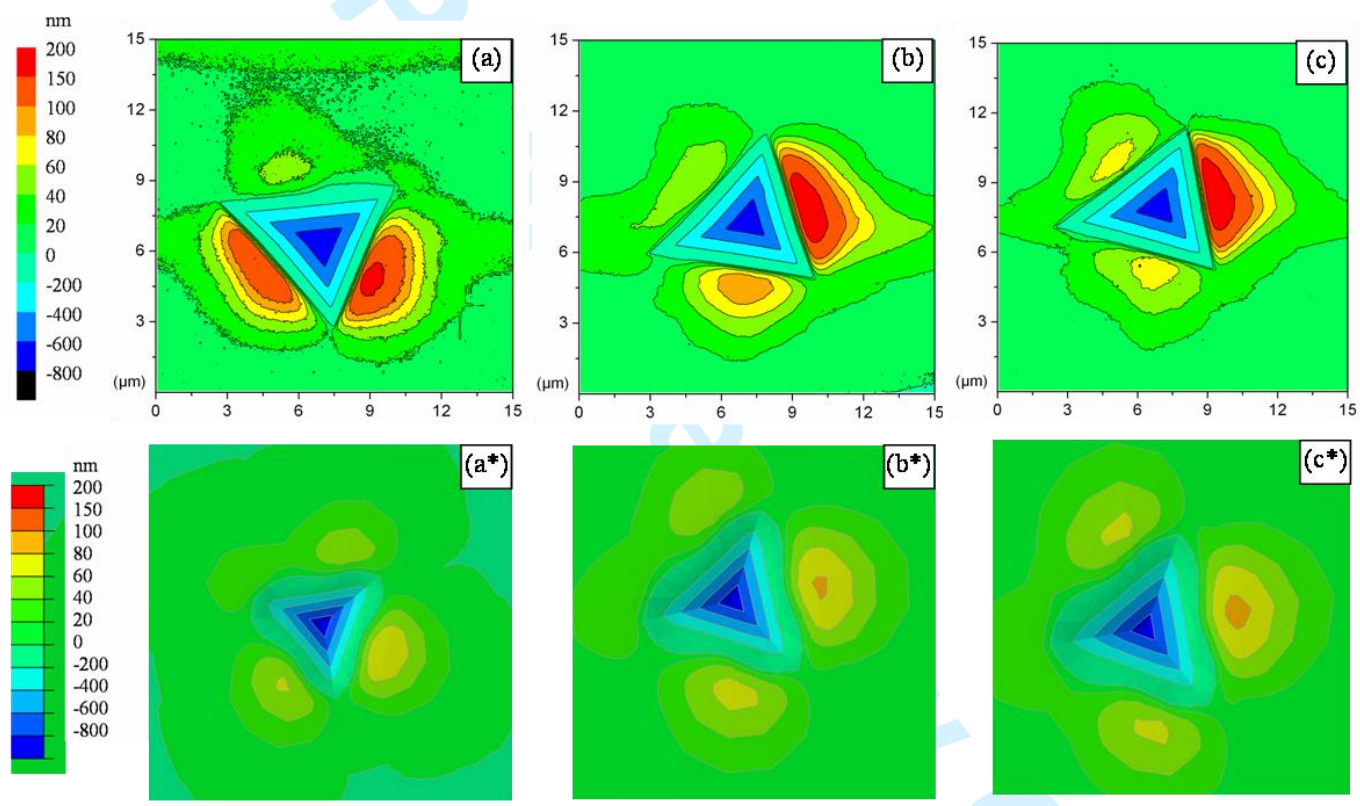

Fig. 5. Surface profiles (after load removal) from Berkovich indentation on (100) plane with in-plane rotated indenter. Experimental results with different rotation angles: (a) $\theta=-20^{\circ}$; (b) $\theta=38^{\circ}$; (c) $\theta=45^{\circ}$; and the corresponding numerical results below are marked with an asterisk $(*)$. 

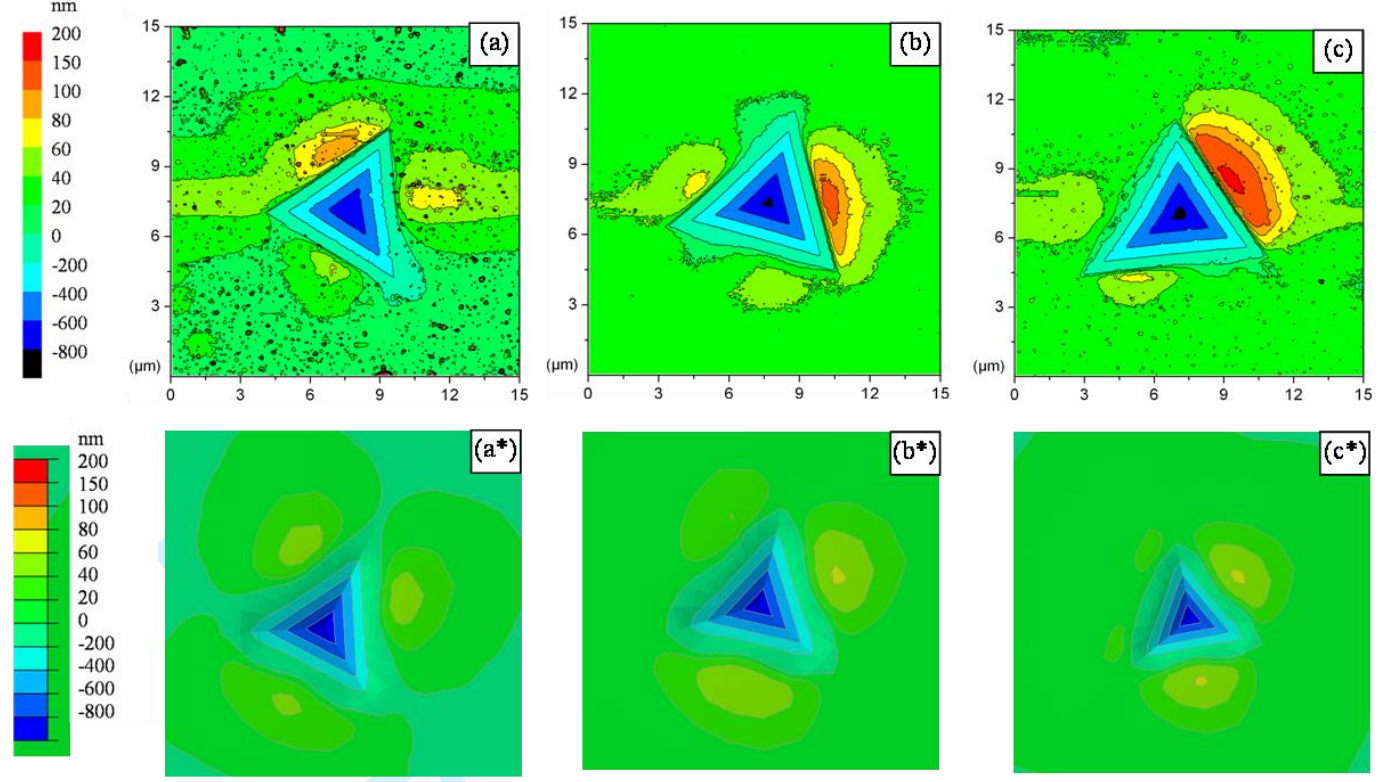

Fig. 6. Surface profiles (after load removal) from Berkovich indentation on (110) plane with in-plane rotated indenter. Experimental results with different rotation angles: (a) $\theta=-90^{\circ}$; (b) $\theta=-45^{\circ}$; (c) $\theta=60^{\circ}$; and the corresponding numerical results below are marked with an asterisk (*).
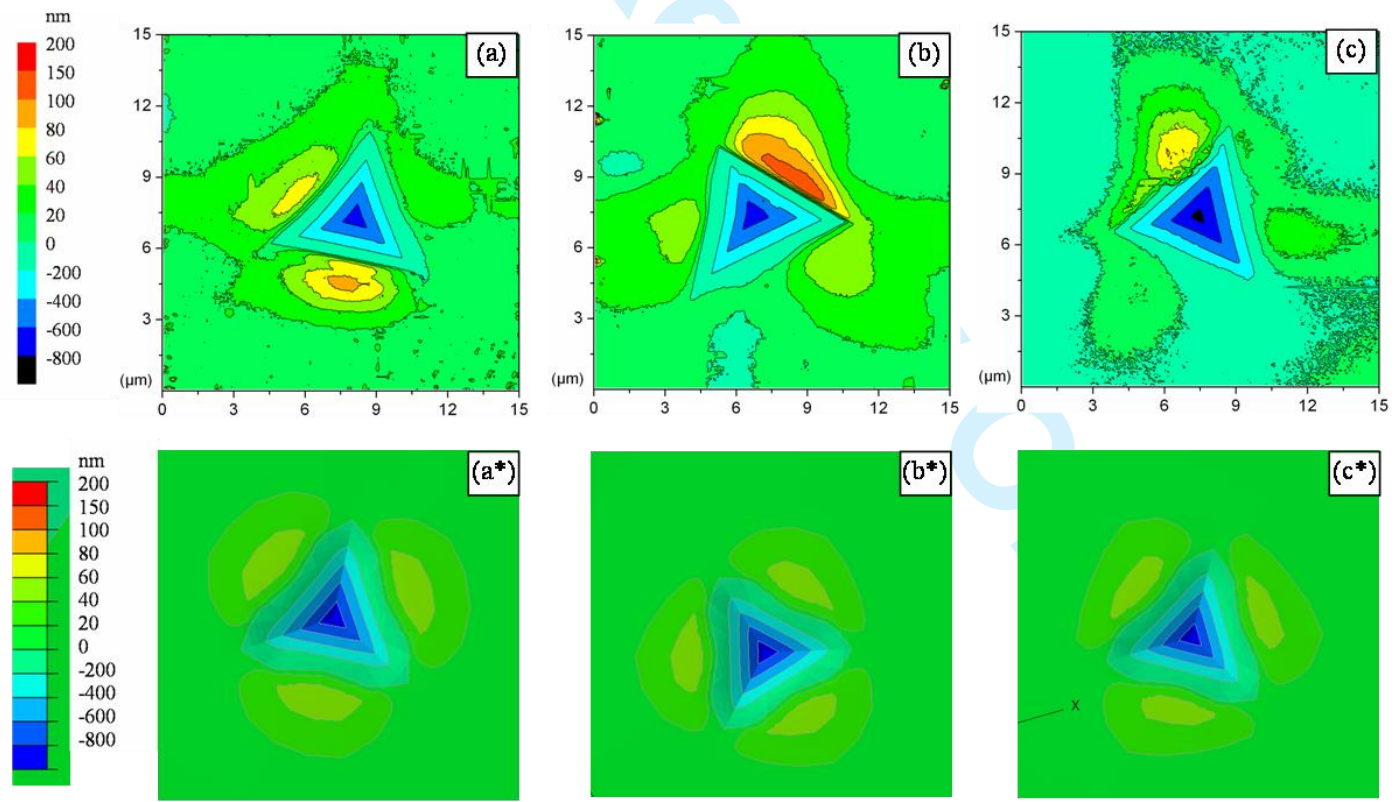

Fig. 7. Surface profiles (after load removal) from Berkovich indentation on (111) plane with in-plane rotated indenter. Experimental results with different rotation angles: (a) $\theta=-100^{\circ}$; (b) $\theta=60^{\circ}$; (c) $\theta=110^{\circ}$; and the corresponding numerical results below are marked with an asterisk $(*)$. 
The measured pile-up patterns seem difficult to interpret probably due to the mismatch between the discrete rotational symmetry of the contact loads and the intrinsic crystal symmetry of specimen surfaces. The inherently preferred slip directions in tungsten belong to the $<111>$ family. These directions are projected as [011], [0 $\overline{1} \overline{1}]$, [01 $\overline{1}]$ and $[0 \overline{1} 1]$ directions onto the $(100)$ plane, as [1 $\overline{1} 1]$ and [ $\overline{1} 11]$ directions onto the (110) plane and as [1 $\overline{2} 1],[\overline{2} 11]$ and [11 $\overline{2}]$ directions onto the (111) plane[45]. If slip occurs under an indentation load in one of these directions without any kinematic constraint, it would produce a pile-up pattern with either 4-, 2- or 3-fold rotational symmetry on the $(100),(110)$ or (111) surface plane, respectively. This feature was already manifested both experimentally and theoretically in the spherical indentation study of tungsten mentioned above [13].

In a Berkovich indentation test with discrete rotational symmetry of contact loads, however, plastic slip in certain preferred directions can be possibly hampered by the polygonal side edges of the indenter. This constraint necessarily affects the original symmetry of the pile-up patterns. As is seen in Fig. 5, there are only three hillocks formed on the (100) surface while the original four-fold symmetry of the pile-up pattern is essentially modified by the triangular edges of the indenter.

On the (100) plane with the hoop orientation of $\theta=-20^{\circ}$ (Fig. 5a), there are two large and one small hillocks next to the edges of the imprint. Both experimental and numerical results show that more material is piled up along the edges of the triangle that are perpendicular to the $[09 \overline{2}]$ and $[0 \overline{3} 8]$ direction on the plane (100) and the smaller pile-up is on the other side of the triangle. For the rotation angle $\theta=38^{\circ}$ shown in Fig. $5 \mathrm{~b}$ the largest hillock is along the edge of the triangle which is perpendicular to the [012] direction and the other hillocks are much smaller. When the rotation angles 
are set $38^{\circ}$ and $45^{\circ}$ (Fig. 5b-c), the pile up patterns becomes very similar. In both cases there is one large and two small hillocks along the edges of the triangles. The large hillocks are formed along the edges perpendicular to the [0 45] and [011] direction, respectively. The [011] direction is the projection of the [111] slip direction onto the (100) plane and the [045] direction is very close to the [011] direction. The only difference between the two pile-up patterns is the height of the small hillocks. For $\theta=38^{\circ}$ the height of two small hillocks is rather different while two small hillocks have almost equal heights for $\theta=45^{\circ}$. The numerical results reproduce the pile-up patterns which catch the variation of the two small hillocks when the indenter is rotated by a small angle $\left(\Delta \theta=7^{\circ}\right)$.

Fig. 6 and Fig. 7 show that the pile-up patterns on the (110) and the (111) plane exhibit a strong dependence on the azimuthal orientation as well. It should be pointed out that the discrepancy between experimental and numerical patterns regarding the pile-up amplitude was relatively large on (110) and (111) planes. The discrepancy could be caused by the deviation of crystal plasticity parameters from the "exact values", since the pile-up amplitude was not considered in the procedure of parameters determination. Apparently, it's necessary to fit both numerical load-displacement curves and pile-up patterns with the experimental counterparts to obtain more accurate crystal plasticity parameters[46]. It's much more complicated to fit the pile-up patterns than to fit simply load-displacement curves, especially when the distribution of pile-ups was not symmetrical here. Nevertheless, the next step would be determination of parameters by fitting both loading curves and the actual shape of the surface pile-ups.

\subsection{The role of the indenter shape}

From the previous section it turned out that the pile-up patterns were sensitively related 
to the azimuthal orientation of the Berkovich indenter with respect to the specimen's crystal orientation. According to the experimental work of Stelmashenko et al using Vickers micro-hardness test [47], however, the distribution of hillocks on tungsten single crystals was hardly dependent on the azimuthal orientation of the indenter. They argued that the pile-up formation was essentially determined by the crystallographic feature of the sample material rather than the stress fields generated by the Vickers indentation. However, a detailed inspection into the pile-up patterns reveals that, even in the Vickers indentation, the position and the size of the hillocks were significantly modified, when the azimuthal orientation of the indenter was varied. For example, when the diagonal of the square-shaped Vickers imprint was oriented along the [0 01$]$ direction, four hillocks were along the four edges which were perpendicular to the preferred $<011>$ slip directions, whereas, when the diagonal of the imprint was oriented along the [011] direction, the four hillocks were formed as well, but not necessarily in the preferred slip directions. Therefore, the pile-ups are essentially affected by the sharp edges of the Vickers indenter. In the case of Vickers indentation on the (100) and (110) planes, the four-fold symmetry of the indenter matches well with the intrinsic symmetry of the pile-up patterns of tungsten which was observed in the spherical indentation test where the number of hillocks on these planes was equal to the case of Vickers indentation.

On the contrary, as the three-fold symmetry of the Berkovich indenter does not match with the intrinsic symmetry of pile-ups on the (100) and (110) planes, the number of hillocks is not necessarily a pure consequence of the crystal symmetry (see Fig. 6 and Fig. 7). The characteristic asymmetry of the pile-up patterns found in the Berkovich indentation test may provide additional information on the slip mechanisms of tungsten together with the results of spherical indentation tests. 
Fig. 8 shows calculated distributions of accumulated shear strain $\gamma$ for indentation with rotated indenter on three crystallographic planes (100), (110), and (111). Here, accumulated shear strain $\gamma$ is defined as the sum of the absolute value of accumulated shear strain for all the activated slip systems. It is clear that the distributions of accumulated shear strain are not sensitive to crystallographic orientations. However, they are strongly dependent on the azimuthal orientation of the indenter. Take the distributions of accumulated shear strain on (100) plane as an example, when the indenter is rotated from [0 $\overline{3} 8]$ to [011] direction the accumulated shear strain distributed along the edge of the indenter irrespective of the crystallographic orientation. This result can be applied to explain the pile-up patterns which are strongly affected by the azimuthal orientation of the indenter.
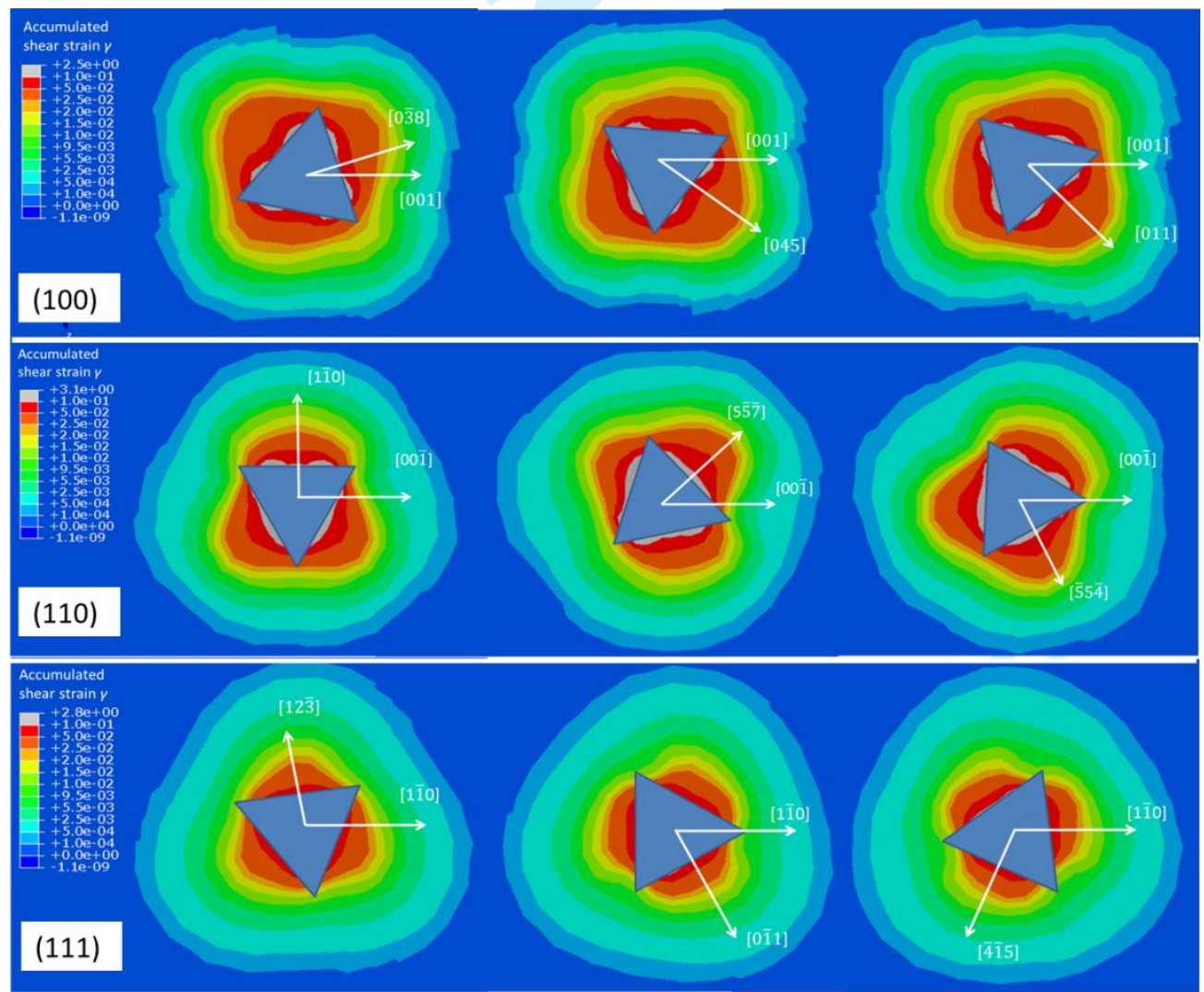

Fig. 8. Accumulated shear strain $\gamma$ contours after unloading from Berkovich indentation on (100), (110), and (111) plane with in-plane rotated indenter. 


\section{Summary and conclusions}

The main findings and conclusions can be summarized as follows:

1. The triangular geometry of the Berkovich imprint dominated the patterns of surface pile-up formation. The height of the pile-up hillocks was often nonuniform even on the same surface plane indicating strong influence of slip geometry leading to plastic anisotropy. The pile-up of material during indentation is a highly characteristic feature of the prevalent crystallographic deformation procedure.

2. Both experimental and numerical results show that the variation of the indenter's azimuthal orientation affects the pile-up patterns. The mismatch of symmetry between the indenter and the indented crystal causes variation of the hillocks' number and height. There is qualitative agreement between the experimental and numerical surface profiles which show the number and height of the hillocks. The agreement for indents on the (100) surface is impressive since the numerical simulations catch the small alteration in height of the hillocks when the difference between the azimuthal orientations of the indenter is as small as $7^{\circ}$

3. The pile-ups formation does not reflect the material's cubic symmetry in Berkovich indentation. The geometrically non-isotropic pyramidal indenter locally breaks the material symmetry of the crystals and cause different pile-up patterns compared with geometrically isotropic spherical indentation. 


\section{Acknowledgements}

One of the authors (W.Z. Yao) is grateful to the Chinese Scholarship Council (CSC) for the financial support.

\section{References}

[1] S.R. Kalidindi, and S. Pathak, Determination of the effective zero-point and the extraction of spherical nanoindentation stress-strain curves, Acta Mater. 56 (2008), pp. 3523-3532.

[2] X. Li, and $\mathrm{B}$. Bhushan, A review of nanoindentation continuous stiffness measurement technique and its applications, Mater. Charact. 48 (2002), pp. 11-36.

[3] Y.J. Park, and G.M. Pharr, Nanoindentation with spherical indenters: finite element studies of deformation in the elastic-plastic transition regime, Thin Solid Films 447-448 (2004), pp. 246-250.

[4] R. Saha, and W.D. Nix, Effects of the substrate on the determination of thin film mechanical properties by nanoindentation, Acta Mater. 50 (2002), pp. 23-38.

[5] H. Bei, Z.P. Lu, and E.P. George, Theoretical strength and the onset of plasticity in bulk metallic glasses investigated by nanoindentation with a spherical indenter, Phys. Rev. Lett. 93 (2004), p. 4.

[6] M.A. Lodes, A. Hartmaier, M. Göken, and K. Durst, Influence of dislocation density on the pop-in behavior and indentation size effect in CaF2 single crystals: Experiments and molecular dynamics simulations, Acta. Mater. 59 (2011), pp. 4264-4273.

[7] W.W. Gerberich, D.E. Kramer, N.I. Tymiak, A.A. Volinsky, D.F. Bahr, and M.D. Kriese, Nanoindentation-induced defect-interface interactions: Phenomena, methods and limitations, Acta Mater. 47 (1999), pp. 4115-4123.

[8] M. Liu, C. Lu, and A.K. Tieu, Crystal plasticity finite element method modelling of indentation size effect, Int. J. Solids Struct. 54 (2015), pp. 42-49.

[9] D. Esque-de los Ojos, J. Ocenasek, and J. Alcala, Sharp indentation crystal plasticity finite element simulations: Assessment of crystallographic anisotropy effects on the mechanical response of thin fcc single crystalline films, Comput. Mater. Sci. 86 (2014), pp. 186-192.

[10] M. Mata, O. Casals, and J. Alcalá, The plastic zone size in indentation experiments: The analogy with the expansion of a spherical cavity, Int.J.Solids Struct. 43 (2006), pp. 59946013.

[11] J. Rodríguez, and M.A.G. Maneiro, A procedure to prevent pile up effects on the analysis of spherical indentation data in elastic-plastic materials, Mech. Mater. 39 (2007), pp. 987-997.

[12] B. Eidel, Crystal plasticity finite-element analysis versus experimental results of pyramidal indentation into (001) fcc single crystal, Acta Mater. 59 (2011), pp. 1761-1771.

[13] W.Z. Yao, C.E. Krill, III, B. Albinski, H.C. Schneider, and J.H. You, Plastic material parameters and plastic anisotropy of tungsten single crystal: a spherical micro-indentation study, J Mater Sci 49 (2014), pp. 3705-3715.

[14] Y. Liu, S. Varghese, J. Ma, M. Yoshino, H. Lu, and R. Komanduri, Orientation effects in nanoindentation of single crystal copper, Int. J. Plast. 24 (2008), pp. 1990-2015.

[15] C. Zambaldi, and D. Raabe, Plastic anisotropy of gamma-TiAl revealed by axisymmetric indentation, Acta Mater. 58 (2010), pp. 3516-3530.

[16] N. Zaafarani, D. Raabe, F. Roters, and S. Zaefferer, On the origin of deformationinduced rotation patterns below nanoindents, Acta Mater. 56 (2008), pp. 31-42.

[17] C.A. Brookes, J.B. O'Neill, and B.A.W. Redfern, Anisotropy in the hardness of single crystals, Proc. R. Soc. London A 322 (1971), pp. 73-88.

[18] J. Alcalá, O. Casals, and J. Ocenásek, Micromechanics of pyramidal indentation in $f c c$ metals: Single crystal plasticity finite element analysis, J.Mech.Phys.Solids 56 (2008), pp. 3277-3303. 
[19] O. Casals, J. Ocenásek, and J. Alcalá, Crystal plasticity finite element simulations of pyramidal indentation in copper single crystals, Acta Mater. 55 (2007), pp. 55-68.

[20] A. Barnoush, Correlation between dislocation density and nanomechanical response during nanoindentation, Acta. Mater. 60 (2012), pp. 1268-1277.

[21] S. Shim, H. Bei, M.K. Miller, G.M. Pharr, and E.P. George, Effects of focused ion beam milling on the compressive behavior of directionally solidified micropillars and the nanoindentation response of an electropolished surface, Acta Mater. 57 (2009), pp. 503-510.

[22] W. Wang, C.B. Jiang, and K. Lu, Deformation behavior of Ni3Al single crystals during nanoindentation, Acta Mater. 51 (2003), pp. 6169-6180.

[23] W.Z. Yao, P. Wang, A. Manhard, C.E. Krill, and J.H. You, Effect of hydrogen on the slip resistance of tungsten single crystals, Mater. Sci. Eng. A 559 (2013), pp. 467-473.

[24] P. Peralta, R. Ledoux, R. Dickerson, M. Hakik, and P. Dickerson, Characterization of surface deformation around vickers indents in monocrystalline materials, Metall. Mater. Trans. A-Phys. Metall. Mater. Sci. 35A (2004), pp. 2247-2255.

[25] N.A. Stelmashenko, and L.M. Brown, Deformation structure of microindentations in W(100): A transmission electron microscopy study, Philos. Mag. A 74 (1996), pp. 11951206.

[26] W.C. Oliver, and G.M. Pharr, An improved technique for determining hardness and elastic modulus using load and displacement sensing indentation experiments, J. Mater. Res. 7 (1992), pp. 1564-1583.

[27] H.J. Chang, M. Fivel, and M. Verdier, Indentation Crystal Plasticity: Experiments and Multiscale Simulations, Mater. Res. Soc. Symp. Proc. 1224 (2009).

[28] D. Peirce, R.J. Asaro, and A. Needleman, An analysis of nonuniform and localized deformation in ductile single crystals, Acta Metall. 30 (1982), pp. 1087-1119.

[29] R.J. Asaro, and J.R. Rice, Strain localization in ductile single crystals, J.Mech.Phys.Solids 25 (1977), pp. 309-338.

[30] R.J. Asaro, and A. Needleman, Flow localization in strain hardening crystalline solids, Scripta Metall. Mater. 18 (1984), pp. 429-435.

[31] R. Hill, Essential structure of constitutive laws for metal composites and polycrystals, J.Mech.Phys.Solids 15 (1967), pp. 79-95.

[32] R. Hill, Generalized constitutive relations for incremental deformation of metal crystals by multislip, J.Mech.Phys.Solids 14 (1966), pp. 95-102.

[33] R. Hill, and J.R. Rice, Constitutive analysis of elastic-plastic crystals at arbitrary strain, J.Mech.Phys.Solids 20 (1972), pp. 401-413.

[34] Y. Huang, A user-material subroutine incorporating single crystal plasticity in the abaqus finite element program, Cambridge,Massachusetts, 1991.

[35] J. Ocenásek, M.R. Ripoll, S.M. Weygand, and H. Riedel, Multi-grain finite element model for studying the wire drawing process, Comput. Mater. Sci. 39 (2007), pp. 23-28.

[36] C. Bohnert, N.J. Schmitt, S.M. Weygand, O. Kraft, and R. Schwaiger, Fracture toughness characterization of single-crystalline tungsten using notched micro-cantilever specimens, Int. J. Plast. 81 (2016), pp. 1-17.

[37] W.D. Nix, Elastic and plastic properties of thin films on substrates: nanoindentation techniques, Mater. Sci. Eng. A 234-236 (1997), pp. 37-44.

[38] Y.B. Park, D.N. Lee, and G. Gottstein, The evolution of recrystallization textures in body centred cubic metals, Acta Mater. 46 (1998), pp. 3371-3379.

[39] Y.-H. Lee, and D. Kwon, Measurement of residual-stress effect by nanoindentation on elastically strained ( 100$) \mathrm{W}$, Scripta Mater. 49 (2003), pp. 459-465.

[40] G. Subhash, Y.J. Lee, and G. Ravichandran, Plastic deformation of CVD textured tungsten--I. Constitutive response, Acta Metall. Mater. 42 (1994), pp. 319-330.

[41] M. Garfinkle, Room-temperature tensile behavior of $\langle 100\rangle$ oriented tungsten single crystals with rehenium in ductile solid solution, Fourth Symposium on Refractory Metals, Indiana, IN,1965.

[42] Y.J. Lee, G. Subhash, and G. Ravichandran, Constitutive modeling of textured bodycentered-cubic (bcc) polycrystals, Int. J. Plast. 15 (1999), pp. 625-645. 
[43] E. Lassner, and W.-D. Schubert, Tungsten: Properties, Chemistry, Technology of the Element, Alloys, and Chemical Compounds, Springer, Berlin, 1999.

[44] W.Z. Yao, Crystal plasticity study of single crystal tungsten by indentation tests. Ph.D.diss., Ulm University, 2012.

[45] L. Kaun, A. Luft, J. Richter, and D. Schulze, Slip line pattern and active slip systems of tungsten and molybdenium single crystals weakly derormed in tension at room temperature, Phys. Stat. Sol. 26 (1968), pp. 485-499.

[46] H.J. Chang, M. Fivel, D. Rodney, and M. Verdier, Multiscale modelling of indentation in FCC metals: From atomic to continuum, C. R. Phys. 11 (2010), pp. 285-292.

[47] N.A. Stelmashenko, M.G. Walls, L.M. Brown, and Y.V. Milman, Microindentations on W and Mo oriented single crystals: An STM study, Acta Metall. Mater. 41 (1993), pp. 28552865. 